\title{
ESCAPE RATE FOR 2-DIMENSIONAL BROWNIAN MOTION CONDITIONED TO BE TRANSIENT AND APPLICATION TO ZYGMUND FUNCTIONS
}

\author{
ELIZABETH ANN HOUSWORTH
}

\begin{abstract}
The escape rate of a 2-dimensional Brownian motion conditioned to be transient is determined to be $P\{X(t)<f(t)$ i.o. as $t \uparrow \infty\}=0$ or 1 according as $\sum_{n=1}^{\infty} e^{-n} \log f\left(e^{e^{n}}\right)<$ or $=\infty$. The result is used to construct a complex-valued Zygmund function-as a lacunary series-whose graph does not have $\sigma$-finite linear Hausdorff measure. This contrasts the result of Mauldin and Williams that the graphs of all real-valued Zygmund functions have $\sigma$-finite linear Hausdorff measure.
\end{abstract}

\section{INTRODUCTION}

A 1-dimensional Brownian motion conditioned not to hit the origin is equivalent to the radial part of a 3-dimensional Brownian motion [11] whose escape rate is known [6]. In other terminology, a Bessel process of index 1 conditioned not to exit $(0, \infty)$ is a Bessel process of index 3. Given the scaling properties of Bessel processes, it can be shown using Durrett's arguments [5, p. 97] and determining the resulting generator that a Bessel process of index $\alpha$ for $1 \leq \alpha<2$ conditioned not to exit $(0, \infty)$ is equivalent to a Bessel process of index $4-\alpha$. The escape rate of Bessel processes has been determined in Shiga and Watanabe [16]. As is noted in [16], the proof is essentially due to Motoo (see, for instance, [12, p. 147] where the proof with integer indices carries over trivially to arbitrary indices).

Since 2-dimensional Brownian motion already does not hit the origin, our theorem gives the escape rate to infinity of 2-dimensional Brownian motion conditioned to be transient by conditioning it not to hit the unit disk. An integral test of the escape rate is given below and the proof is an adaptation of a proof of Dvoretsky and Erdös [6] for the escape rate of Brownian motion in dimensions greater than 2. The arguments of Dvoretsky and Erdös and of Motoo rely on the scaling properties of Brownian motion. These arguments do not apply to the diffusion under consideration here. Attempts to apply these arguments to more general 1-dimensional diffusions have not succeeded beyond the case of Bessel processes discussed by Shiga and Watanabe.

Received by the editors February 8, 1993.

1991 Mathematics Subject Classification. Primary 60J60, 30D40.

Key words and phrases. Brownian motion, killed processes, generators, scale functions, Zygmund functions, lacunary series.

Research supported by NSF Grant DMS 8701212 and ONR Grant N0014-90-J-1639. 
We then apply the escape rate result to the partial sums of the derivative of a lacunary series in the Zygmund class in order to show that the graph does not have $\sigma$-finite linear Hausdorff measure. This provides a complex counterexample to a theorem of Mauldin and Williams [14] asserting that the graphs of all real-valued Zygmund functions do have $\sigma$-finite linear Hausdorff measure.

This work is part of the author's dissertation written under the direction of Loren Pitt who suggested the problem and provided generous guidance. The author also gratefully acknowledges the support of the University of Virginia and its mathematics department during her graduate studies. The analytic counterexample was obtained later and the author also wishes to thank Rodrigo Bañuelos and Burgess Davis for some helpful discussions.

\section{ESCAPE RATE}

Theorem 1. Let $X(t)$ be the radial part of 2-dimensional Brownian motion conditioned to go to $\infty$ by conditioning it to stay away from the closed unit disk, $\mathbb{D}$, up to time $T$ and then letting $T \rightarrow \infty$. The conditioned process, $X(t)$, is the radial part of a $\log \|z\|$-transform of 2-dimensional Brownian motion killed when it hits $\mathbb{D}$. Let $Q^{x}$ be the conditional probability measure associated with $X(t)$ and $X(0)=x>1$. For increasing functions, $f(t) \geq 1, Q^{x}\{X(t)<f(t)$ i.o., $t \uparrow \infty\}=0$ or 1 according as $\sum_{n=1}^{\infty} e^{-n} \log f\left(e^{e^{n}}\right)<$ or $=\infty$.

The theorem implies, for instance, that with probability one, $X(t)$ falls below $\exp \{\log n / \log \log n\}$ infinitely often but not below $\exp \left\{\log n /(\log \log n)^{(1+\varepsilon)}\right\}$ infinitely often.

Recall: A Markov process $Y(t)$ with transition density $q(t, x, y)$ is an $h$ transform of another Markov process $X(t)$ with transition density $p(t, x, y)$ if $q(t, x, y)=(h(x))^{-1} p(t, x, y) h(y)$. See, for reference, Durrett [5, Chapter 3]. From now on, $p(t, x, y)$ will denote the transition density for 2-dimensional Brownian motion, $p_{1}(t, x, y)$ for 2-dimensional Brownian motion killed when it hits $\mathbb{D}$, and $q(t, x, y)$ for 2-dimensional Brownian motion conditioned to stay away from $\mathbb{D}$.

We first show that this conditioning leads to a $\log \|z\|$-transform by arguing similarly to Knight [11, Theorem 3.1]. Let $P^{z}$ denote the probability measure associated with 2-dimensional Brownian motion starting from $z$ and $q_{T}$ the joint densities of 2-dimensional Brownian motion given that $B(t)$ does not hit $\mathbb{D}$ up to time $T$. Then, setting $B(0)=z$, we see that

$$
\begin{aligned}
& q_{T}\left(t_{1}, \ldots, t_{n}, z_{1}, \ldots, z_{n}\right) \\
& \quad=\prod_{j=1}^{n} p_{1}\left(t_{j}-t_{j-1}, z_{j-1}, z_{j}\right) \frac{P^{z_{n}}\left\{\min _{t<T-t_{n}}\|B(t)\|>1\right\}}{P^{z}\left\{\min _{t<T}\|B(t)\|>1\right\}} .
\end{aligned}
$$

Hunt [10] showed that the probability 2-dimensional Brownian motion starting at a radius $r$ does not hit the disk $\mathbb{D}$ by time $T$ is asymptotic to $\{\log T\}^{-1} 2 \pi \log r$ as $T \rightarrow \infty$. Thus, as $T \rightarrow \infty$,

$$
\frac{P^{z_{n}}\left\{\min _{t<T-t_{n}}\|B(t)\|>1\right\}}{P^{z}\left\{\min _{t<T}\|B(t)>1\|\right\}} \rightarrow \frac{\log \left\|z_{n}\right\|}{\log \|z\|}
$$

and $q(t, x, y)=p_{1}(t, x, y) \log \|y\|(\log \|x\|)^{-1}$, as claimed.

For the rest of the proof of the theorem, we make use of the scale function for our diffusion $X(t)$ and the subsequent lemmas. The scale function is a 
strictly increasing function on the state space (in our case $(1, \infty)$ ) so that for $a \leq x \leq b$ in the state space, the probability starting from $x$ that $X(t)$ hits $b$ before $a$ is equal to $\{s(x)-s(a)\} /\{s(b)-s(a)\}$.

The scale function also satisfies $G s(x)=0$ where $G$ is the infinitesimal generator for the diffusion (see, for reference, Knight [12, $\mathrm{p}_{\mathrm{i}}$ 176]). The infinitesimal generator for the radial part of 2-dimensional Brownian motion killed when it hits $\mathbb{D}$ is well known to be $G f(x)=f^{\prime \prime}(x) / 2+f^{\prime}(x) /(2 x)$ for bounded, continuous, twice differentiable functions $f$ on $(1, \infty)$. Since $X(t)$ is a $\log x$-transform of this process, the generator for $X$ is

$$
\begin{aligned}
G f(x) & =\frac{1}{\log x}\left(\frac{1}{2}(f(x) \log x)^{\prime \prime}+\frac{1}{2 x}(f(x) \log x)^{\prime}\right) p \\
& =\frac{1}{2} f^{\prime \prime}(x)+\left\{\frac{1}{2 x}+\frac{1}{x \log x}\right\} f^{\prime}(x) .
\end{aligned}
$$

Thus it is easy to see that $s(x)=-1 / \log x$ satisfies the requirements for the scale function.

Lemma 1. For $x>r, Q^{x}\{X(t)<r$ for some $t>0\}=\log r / \log x$.

Proof.

$$
\begin{gathered}
Q^{x}\{X(t)<r \text { some } t>0\}=\lim _{b \rightarrow \infty} Q^{x}\{X(t) \text { hits } r \text { before } b\} \\
=\lim _{b \rightarrow \infty} \frac{s(b)-s(x)}{s(b)-s(r)}=\frac{\log r}{\log x} .
\end{gathered}
$$

Lemma 2. Assuming $T>1$ and $1<x<T / 2$,

$$
\begin{aligned}
\frac{\log r}{\log T}-o\left(T^{-.1}\right) / \log x & \leq Q^{x}\{X(t)<r \text { some } t>T\} \\
& \leq \frac{2.5 \log r}{\log T}+o\left(T^{-.1}\right) / \log x .
\end{aligned}
$$

Remark. The constant 2.5 fits both the needs of the proof below for a constant larger than 2 and the needs of later applications for a constant less than $e$.

Proof. For the upper bound, conditioning on whether $X(T)<T^{2 / 5}$ or not, $Q^{x}\{X(t)<r$ for some $t>T\}$ is smaller than

$$
Q^{x}\left\{X(T)<T^{2 / 5}\right\}+Q^{x}\left\{X(t)<r \text { some } t>T \text { and } X(T)>T^{2 / 5}\right\} .
$$

However, $Q^{y}\{X(t)<r$ some $t>0\}$ is a decreasing function of $y$. Thus

$$
\begin{aligned}
Q^{x}\{X(t)<r \text { some } t>T\} \leq & Q^{T^{2 / 5}}\{X(t)<r \text { some } t>0\} \\
& +Q^{x}\left\{X(T)<T^{2 / 5}\right\} \\
\leq & \frac{2.5 \log r}{\log x}+Q^{x}\left\{X(T)<T^{2 / 5}\right\} .
\end{aligned}
$$

The lower bound is obtained by conditioning on whether $X(T)>T$ or not. Thus $Q^{x}\{X(t)<r$ some $t>T\}$ is greater than

$$
Q^{T}\{X(t)<r \text { some } t>0\}-Q^{x}\{X(T)>T\} \geq \frac{\log r}{\log T}-Q^{x}\{X(T)>T\} .
$$


We now estimate

$$
\begin{aligned}
Q^{x}\left\{X(T)<T^{2 / 5}\right\} & \leq \int_{\left\{1<\|z\|<T^{2 / 5}\right\}} p_{1}(T, x, z) \frac{\log \|z\|}{\log x} d z \\
& \leq \frac{\log T^{2 / 5}}{\log x} \int_{\left\{\|z\|<T^{2 / 5}\right\}} \frac{1}{2 \pi T} \exp \left\{\frac{-\|z-x\|^{2}}{2 T}\right\} d z .
\end{aligned}
$$

Since the integral becomes larger when made symmetric around the origin, we can estimate instead the larger

$$
\frac{\log T^{2 / 5}}{\log x} \int_{\left\{\|z\|<T^{2 / 5}\right\}} \frac{1}{2 \pi T} \exp \left\{\frac{-\|z\|^{2}}{2 T}\right\} d z \leq \frac{\pi T^{4 / 5} \log T^{2 / 5}}{2 \pi T \log x}=\frac{o\left(T^{-.1}\right)}{\log x} .
$$

Similarly,

$$
\begin{aligned}
Q^{x}\{X(T)>T\} & =\int_{\{\|z\|>T\}} p_{1}(T, x, z) \frac{\log \|z\|}{\log x} d z \\
& \leq \int_{\{\|z\|>T\}} \frac{\log \|z\|}{\log x} \frac{1}{2 \pi T} \exp \left\{\frac{-\|z-x\|^{2}}{2 T}\right\} d z .
\end{aligned}
$$

Since $x<T / 2$, we can consider the larger integral

$$
\int_{\left\{\|z\|>\frac{1}{2} T\right\}} \frac{\log 2\|z\|}{\log x} \frac{1}{2 \pi T} \exp \left\{\frac{-\|z\|^{2}}{2 T}\right\} d z=\frac{o\left(T^{-.1}\right)}{\log x} .
$$

With these estimates we can complete the proof of Theorem 1. To ease notation, let $e_{n}=e^{e^{n}}$.

In one direction, assume $\sum e^{-n} \log f\left(e_{n}\right)<\infty$. Let $A_{n}$ be the event that $X(t)<f(t)$ for some $t$ in the interval $\left(e_{n}, e_{n+1}\right)$. Then

$$
\begin{aligned}
Q^{x}\left(A_{n}\right) & \leq Q^{x}\left\{X(t)<f\left(e_{n+1}\right) \text { some } t>e_{n}\right\} \\
& \leq \frac{2.5 \log f\left(e_{n+1}\right)}{e^{n}}+o\left(e_{n}^{-.1}\right) / \log x
\end{aligned}
$$

which sums. By the standard Borel-Cantelli theorem, we may now conclude that $Q^{x}\left(A_{n}\right.$ i.o. $)=0$.

The other direction is more complicated since the $A_{n}$ 's are not independent. We make use of a generalized Borel-Cantelli theorem due to Kochen and Stone [13]. We verify that if $\sum e^{-n} \log f\left(e_{n}\right)=\infty$, then

(1) $\sum Q^{x}\left(A_{n}\right)=\infty$ and

(2) $Q^{x}\left(A_{j} A_{k}\right)<c Q^{x}\left(A_{j}\right) Q^{x}\left(A_{k}\right)$ for an absolute constant $c$ and all $k>$ $j+1$.

We can then conclude that $Q^{x}\left(A_{n}\right.$ i.o. $)>0$. Applying a remark of Durrett [5, p. 104], will show that we have a 0-1 law for our conditioned process giving $Q^{x}\left(A_{n}\right.$ i.o. $)=1$.

First we show that $\sum Q^{x}\left(A_{n}\right)=\infty$, which follows from Lemma 2, since

$$
\begin{aligned}
Q^{x}\left(A_{n}\right) \geq & Q^{x}\left\{X(t)<f\left(e_{n}\right) \text { some } t \in\left(e_{n}, e_{n+1}\right)\right\} \\
\geq & Q^{x}\left\{X(t)<f\left(e_{n}\right) \text { some } t>e_{n}\right\} \\
& -Q^{x}\left\{X(t)<f\left(e_{n}\right) \text { some } t>e_{n+1}\right\} .
\end{aligned}
$$

Using Lemma 2 on both terms yields the lower bound

$$
\left(1-\frac{2.5}{e}\right) \frac{\log f\left(e_{n}\right)}{e^{n}}-o\left(e_{n}^{-.1}\right) / \log x
$$


so $\sum Q^{x}\left(A_{n}\right)=\infty$. Note: this is where we needed the constant in Lemma 2 to be chosen less than $e$.

Next we show for $k>j+1, Q^{x}\left(A_{j} A_{k}\right) \leq c \cdot Q^{x}\left(A_{j}\right) Q^{x}\left(A_{k}\right)$ which also follows from Lemma 2 , since $Q^{x}\left(A_{j} A_{k}\right)$ is smaller than

$Q^{x}\left\{X(t)<f\left(e_{j+1}\right)\right.$ some $t \in\left(e_{j}, e_{j+1}\right)$ and $X(t)<f\left(e_{k+1}\right)$ some $\left.t \in\left(e_{k}, e_{k+1}\right)\right\}$.

Conditioning on whether at time $T=\exp [\exp ([k+j] / 2)], X(T)>T^{2 / 5}$ or not and estimating similar to above, we see that this is smaller than

$$
\begin{aligned}
Q^{x}\{ & \left.X(t)<f\left(e_{j+1}\right) \text { some } t>e_{j}\right\} \\
& \times Q^{T^{2 / 5}}\left\{X(t)<f\left(e_{k+1}\right) \text { some } t>e_{k}-T\right\}+Q^{x}\left\{X(T)<T^{2 / 5}\right\} \\
\leq & \left\{\frac{2.5 \log f\left(e_{j+1}\right)}{e^{j}}+\frac{o\left(e_{j}^{-.1}\right)}{\log x}\right\} \\
& \times\left\{\frac{2.5 \log f\left(e_{k+1}\right)}{\log \left(e_{k}-T\right)}+\frac{o\left(\left\{e_{k}-T\right\}^{-.1}\right.}{\log x}\right\}+\frac{o\left(T^{-.1}\right)}{\log x} .
\end{aligned}
$$

Since $e_{k}-T=e_{k}\left(1-\exp \left\{e^{(k+j) / 2}-e^{k}\right\}\right) \geq e_{k}(1-1 / e)$, we see that,

$$
Q^{x}\left(A_{j} A_{k}\right) \leq 2 \frac{(2.5)^{2} e \log f\left(e_{j+1}\right) \log f\left(e_{k+1}\right)}{(e-1) e^{j} e^{k}} .
$$

We know via the proof that $\sum Q^{x}\left(A_{n}\right)=\infty$ above that

$$
Q^{x}\left(A_{j}\right) \geq(1-2.5 / e) e^{-j} \log f\left(e_{j}\right)-o\left(e_{j}^{-.1}\right) / \log x .
$$

We assume that $f(t) \leq t$ so that replacing $f\left(e_{j}\right)$ with $f\left(e_{j+1}\right)$ in this inequality poses at most a constant multiple change. If $f(t)>t$, then since $X(t)$ will fall below $t$ infinitely often for large $t$, it will fall below $f(t)$ too. The other terms are small in comparison to $Q^{x}\left(A_{j}\right) Q^{x}\left(A_{k}\right)$. Consequently, $Q^{x}\left(A_{j} A_{k}\right) \leq$ $c Q^{x}\left(A_{j}\right) Q^{x}\left(A_{k}\right)$.

By the generalized version of the Borel-Cantelli theorem in Kochen and Stone [13], this is enough to conclude that $Q^{x}\left\{A_{j}\right.$ i.o. $\}>0$. However, we have a $0-1$ law for our process according to the remark in Durrett [5, p. 104] as long as we know that whenever $0 \leq u(z) \leq \log \|z\|, u(z)$ harmonic in $\mathbb{C} \backslash \mathbb{D}$, then $u(z)=c \log \|z\|$. We know that $u\left(r e^{i \theta}\right)=\sum_{-\infty}^{\infty}\left\{a_{n} r^{n}+\overline{a_{-n}} r^{-n}\right\} e^{i n \theta}+c \log r$ (see, for instance, Axler [4, p. 248], The Logarithm Conjugation Theorem). So $-c \log r \leq \sum_{-\infty}^{\infty}\left\{a_{n} r^{n}+\overline{a_{-n}} r^{-n}\right\} e^{i n \theta} \leq(1-c) \log r$ and integrating against $e^{-i n \theta}$ on $[-\pi, \pi]$ to pull off the $n$th coefficient yields for each $n,-c \log r \leq$ $\left\{a_{n} r^{n}-\overline{a_{-n}} r^{-n}\right\} \leq(1-c) \log r$. For $n \neq 0$ this gives us $a_{n}=0$, since $r^{n}$ for positive $n$ and $r^{-n}$ for negative $n$ grow more rapidly than $\log r$. Then the only constant $a_{0}+\overline{a_{0}}$ satisfying $-c \log r \leq a_{0}+\overline{a_{0}} \leq(1-c) \log r$ for all $r>1$ is 0 .

\section{APPLICATION TO GRAPHS OF ZYGMUND FUNCTIONS}

The Zygmund space, $\Lambda^{*}$, consists of continuous functions with period 1 satisfying the smoothness requirement:

$$
\sup _{x, h}\left|\frac{f(x+h)+f(x-h)-2 f(x)}{2 h}\right| \equiv\|f\|_{*}<\infty .
$$


Such functions may still be nondifferentiable. For reference, see [3]. The analytic Zygmund space, $\Lambda_{a}^{*}$, consists of complex-valued Zygmund functions with analytic extensions inside the unit disk (the disk being parameterized by $e^{2 \pi i x}$, $x \in[0,1])$. An important subclass of Zygmund functions consists of lacunary series of the form

$f(x)=\sum_{k=0}^{\infty} \frac{a_{k}}{n_{k}} e^{2 \pi i n_{k} x} \quad$ for which $\inf _{k} \frac{n_{k+1}}{n_{k}} \equiv q>1, n_{0} \geq 1$, and $\left\|a_{k}\right\|_{\infty}<\infty$.

Recall that Hausdorff measure, $m_{h}$ is defined as

$$
m_{h}(A)=\liminf _{\varepsilon \rightarrow 0}\left\{\sum h\left\{\operatorname{diam}\left(B_{n}\right)\right\}: A \subset \bigcup B_{n}, \operatorname{diam}\left(B_{n}\right)<\varepsilon\right\}
$$

and that linear Hausdorff measure is determined by $h(x)=x$ and is denoted by $m_{1}$.

Mauldin and Williams [14] have shown that the graph of all real-valued Zygmund functions have $\sigma$-finite linear Hausdorff measure. Anderson and Pitt [3, Theorem 6.4] proved this result by considering the behavior of the difference quotients. The problem in covering the graph occurs above the set where the difference quotients tend to infinity. In the real case, they tend to positive infinity or to negative infinity but not to both. In the complex case, the difference quotients may spiral to infinity. This means the graph twists and thus is "thicker" than a line. Even though this occurs only above a set of zero Lebesgue measure, the graph above this set need not have $\sigma$-finite linear Hausdorff measure. Indeed,

Theorem 2. The graph of $f(x)=\sum_{k=0}^{\infty} e^{2 \pi i n_{k} x} / n_{k}$ where $n_{k}=2^{2^{k}}$ does not have $\sigma$-finite linear Hausdorff measure.

We will apply a theorem of Rogers and Taylor [15, Theorem 2], which implies that if we construct a measure $\nu$ on the graph of $f$ so that

$$
\frac{\nu(\operatorname{Ball}[(x, f(x)), \varepsilon])}{\varepsilon} \rightarrow 0 \quad \text { as } \varepsilon \rightarrow 0 \nu \text {-a.e. }
$$

then the graph does not have $\sigma$-finite linear Hausdorff measure. We begin by showing that the $N$ th partial sum of the derivative of $f /(2 \pi i), \sum_{k=0}^{N} e^{2 \pi i n_{k} x}$, approximates the symmetric difference quotient and looks very much like 2dimensional Brownian motion at time $N$. The measure $\nu$ will be the projection onto the graph of $f$ of our conditional probability measure of the last section, $Q$, on the probability space $[0,1]$.

Lemma 3. There exists a sequence of random variables $\theta_{k}$ uniformly distributed on $[0,1]$ and defined on some probability space $\Omega$ and a measure theoretic isomorphism $T: \Omega \rightarrow[0,1]$ so that $\left|e^{2 \pi i n_{k} x}-e^{2 \pi i \theta_{k}\left(T^{-1} x\right)}\right| \leq 4 \pi / n_{k}$ for all $x$. Proof. The proof is a slight modification of Hawkes (see Theorem 1 of [8]). We write $x$ in its diadic expansion, $x=x_{1} x_{2} x_{3} \ldots$. The choice for the form of the diadics is unimportant. Let $x(k)$ denote the truncation $x_{1} x_{2} \cdots x_{2^{k}}$. Note that $e^{2 \pi i n_{k} x(k+1)}$ depends only on the $2^{k}$ through $2^{k+1}$ terms of the diadic expansion of $x$. Therefore, $\left\{Y_{k}(x)\right\} \equiv\left\{e^{2 \pi i n_{k} x(k+1)}\right\}$ are independent random variables over $[0,1]$ which approximate $\left\{e^{2 \pi i n_{k} x}\right\}$, i.e. $\left|e^{2 \pi i n_{k} x}-e^{2 \pi i n_{k} x(k+1)}\right| \leq$ $2 \pi n_{k} / n_{k+1}=2 \pi / n_{k}$. 
We can approximate $\left\{n_{k} x(k+1)\right\}$ by independent, uniform random variables. The proof follows directly from Hawkes [8, Theorem 1, p. 24], where his $\beta_{k}$ correspond to our $n_{k} x(k+1)$ and his $N_{k}$ correspond to our $n_{k+1}$. We include the argument for completeness.

Consider independent random variables $\left\{\phi_{k}\right\}$ each uniformly distributed over $[0,1]$. Let $\theta_{k}=\left(n_{k+1} \phi_{k}\right)$ where $(\cdot)$ denotes taking fractional part. Then $\left\{\theta_{k}\right\}$ are also independent and uniformly distributed over $[0,1]$. Let $\gamma_{k}=$ $\left[n_{k+1} \phi_{k}\right] \bmod n_{k}$ where [.] denotes taking the integer part. Then $\left|\gamma_{k} / n_{k}-\theta_{k}\right| \leq$ $1 / n_{k}$ and $\left\{\gamma_{k} / n_{k}\right\}$ have the same joint distributions as $n_{k} x(k+1)$. Thus there exists a measure theoretic isomorphism, $T$, from the probability space supporting independent, identically distributed uniform random variables, $\left\{\theta_{k}\right\}$, to $[0,1]$ so that $\left|e^{2 \pi i n_{k} x(k+1)}-e^{2 \pi i \theta_{k}\left(T^{-1} x\right)}\right| \leq 2 \pi / n_{k}$.

The random variables, $\left\{e^{2 \pi i \theta_{k}}\right\}$, can be embedded into 2-dimensional Brownian motion, $B(t, \omega)$, in the standard fashion. That is, we introduce stopping times $\tau_{1}(\omega), \tau_{2}(\omega), \ldots$ so that $\tau_{1}=\inf \{t>0:|B(t)-B(0)|=1\}$ and $\tau_{j}=\inf \left\{t>\tau_{j-1}:\left|B(t)-B\left(\tau_{j-1}\right)\right|=1\right\}$. Since $B\left(\tau_{k}\right)-B\left(\tau_{k-1}\right)$ is uniformly distributed on the unit circle, we can introduce another measure theoretic isomorphism, $S$, from the probability space supporting the Brownian motion to $[0,1]$ so that $B\left(\tau_{k}, S^{-1} x\right)-B\left(\tau_{k-1}, S^{-1} x\right)=e^{2 \pi i \theta_{k}\left(T^{-1} x\right)}$ and $\left|B\left(\tau_{k}, S^{-1} x\right)-B\left(0, S^{-1} x\right)-\sum_{j=1}^{k} e^{2 \pi i n_{j} x}\right| \leq 4 \pi$. This implies that we can compare the probabilities that Brownian motion and our partial sums stay away from disks by the following inclusions:

$$
\begin{aligned}
& \left\{x:\left|B\left(t, S^{-1} x\right)\right|>12 \pi \text { for all } t<\tau_{n} \text { given } B(0)=100\right\} \\
& \quad \subset\left\{x:\left|100+\sum_{j=1}^{k}\left(e^{2 \pi i n_{j} x}\right)\right|>8 \pi \text { for all } k \leq n\right\} \\
& \\
& \quad \subset\left\{x:\left|B\left(\tau_{k}, S^{-1} x\right)\right|>4 \pi \text { for all } k \leq n \text { given } B(0)=100\right\} \\
& \quad \subset\left\{x:\left|B\left(t, S^{-1} x\right)\right|>3 \pi \text { for all } t \leq \tau_{n} \text { given } B(0)=100\right\} .
\end{aligned}
$$

In addition, the proof of the weak law of large numbers (see, for instance, [7, p. 235]) shows that $P\left\{\left|\tau_{n} / n-1\right|>1 / 2\right\}=o(1 / \log n)$.

Together, these imply that conditioning the partial sums, $\left|100+\sum_{j=1}^{k}\left(e^{2 \pi i n_{j} x}\right)\right|$, to stay way from $8 \pi$ induces a probability measure $Q$ on $[0,1]$, singular with respect to Lebesgue measure, for which the partial sums to go to infinity with $Q$-probability 1 . The probability that Brownian motion has stayed away from the disk of radius $8 \pi$ up to time $N$ starting from 100 is approximately $\log N / \log 100$. The behavior of the $k$ th partial sum of $f^{\prime}(x)$ is the same at all the points in the diadic interval of length $1 / n_{N}$ for all $k \leq N$. Thus, the conditioning weights the intervals of length $1 / n_{N}$ on which the partial sums have stayed away from the disk of radius $8 \pi$ up to time $N$ by approximately $\log N / \log 100$. That is, there exists constants $c, C$ so that

$$
c \frac{\log 100}{\log N} \leq \mid\left\{x:\left|100+\sum_{j=1}^{k}\left(e^{2 \pi i n_{j} x}\right)\right|>8 \pi \text { for all } k \leq N\right\} \mid \leq C \frac{\log 100}{\log N} .
$$

We will make use of the following regularity condition on $Q$. Let $I_{m}(k)$ denote the interval $\left[k / n_{m},(k+1) / n_{m}\right)$. Also let $S_{k}(x)=\sum_{j=1}^{k}\left(e^{2 \pi i n_{j} x}\right)$. 
Lemma 4. $Q\left(I_{m}(k)\right) \leq C\left(n_{m}\right)^{-1}(\log m)$.

Proof. For $M>m^{2}$, the conditioning up to time $M$ yields a conditional probability measure $Q_{M}$ on $[0,1]$ and

$$
Q_{M}\left(I_{m}(k)\right)=\frac{\mid\left\{x \in I_{m}(k):\left|100+S_{j}(x)\right|>8 \pi \text { for all } j \leq M\right\} \mid}{\mid\left\{x \in[0,1]:\left|100+S_{j}(x)\right|>8 \pi \text { for all } j \leq M\right\} \mid} .
$$

The proportion of $x$ in $I_{m}(k)$ such that $\left|100+S_{j}\right|>8 \pi$ for all $j \leq m$ is zero if $\left|100+S_{j}\right| \leq 6 \pi$ for any $j<m$ and any $x \in I_{m}(k)$. If $\left|100+S_{j}\right|>6 \pi$ for all $j<m$ and all $x \in I_{m}(k)$ then the proportion of $x$ in $I_{m}(k)$ so that $\left|100+S_{j}\right|>6 \pi$ for all $j \leq M$ is the same as the proportion in $[0,1]$ so that $\left|100+S_{j}\right|>6 \pi$ for all $j \leq M-m$ given the same starting point. Note that $\left|S_{m}(x)\right| \leq m$. Thus $Q_{M}\left(I_{m}(k)\right)$ is less than or equal to

$$
\begin{gathered}
\frac{\left(n_{m}\right)^{-1} \mid\left\{x \in[0,1]:\left|m+100+S_{j}(x)\right|>6 \pi \text { for all } j \leq M-m\right\} \mid}{c \log 100 / \log M} \\
\quad \leq C^{\prime}\left(n_{m}\right)^{-1} \log M \frac{\log (100+m)}{\log (M-m)} \leq C^{\prime \prime}\left(n_{m}\right)^{-1}(\log m) .
\end{gathered}
$$

We also need to know that these partial sums approximate the difference quotients of our original function, $f$, as given in Theorem 2 .

Lemma 5. There exists a constant $K$ so that for all $h \in\left[1 / n_{k+1}, 1 / n_{k}\right], k>1$,

$$
\left|S_{k}(x)-\frac{f(x+h)-f(x-h)}{2 h}\right| \leq K .
$$

Proof. This is a straightforward combination of Proposition A in [1] and the proof of Theorem 1 in [2]. Consider $f$ as a function in the unit disk, i.e. $f(z)=\sum_{k=0}^{\infty} z^{n_{k}} / n_{k}$. Proposition A in [1] says

$$
\sup _{\theta, h}\left|r e^{2 \pi i \theta} f^{\prime}\left(r e^{2 \pi i \theta}\right)-\frac{f\left(e^{2 \pi i(\theta+h)}\right)-f\left(e^{2 \pi i(\theta-h)}\right)}{4 \pi i h}\right| \leq K_{1},
$$

for $r=1-2 h$ and $h<1 / 2$.

Following the proof of Theorem 1 in [2, p. 70], switching from the right half-plane to the unit disk, we see that, for $h \in\left[1 / n_{k+1}, 1 / n_{k}\right]$ and $r=1-2 h$,

$$
\begin{aligned}
& \left|r e^{2 \pi i \theta} f^{\prime}\left(r e^{2 \pi i \theta}\right)-S_{k}(\theta)\right| \leq \sum_{j=1}^{k}\left(1-r^{n_{j}}\right)+\sum_{j=k+1}^{\infty} r^{n_{j}} \\
& \quad \leq \sum_{j=1}^{k}\left(1-\left(1-1 / n_{k}\right)^{n_{j}}\right)+\sum_{j=k+1}^{\infty}\left(1-1 / n_{k+1}\right)^{n_{j}} \\
& \quad \leq \sum_{j=1}^{k} \frac{n_{j}}{n_{k}}+\sum_{j=k+1}^{\infty}\left(\left(1-1 / n_{k+1}\right)^{n_{k+1}}\right)^{n_{j} / n_{k+1}} \\
& \quad<\infty
\end{aligned}
$$

for any lacunary series and in particular for $n_{k}=2^{2^{k}}$.

Proof of Theorem 2. Anderson and Pitt [3] have shown that the graph of $f$ above an interval $I=[a, b]$ is contained in a cylinder about the line connecting $(a, f(a))$ to $(b, f(b))$ of radius $2\|f\|_{*}|I|$. For our function, $\|f\|_{*}=1$. 
We now wish to apply the theorem of Rogers and Taylor. Let $G(f)$ denote the graph of $f$, i.e. $\{(x, f(x)): x \in[0,1]\}$. We define $\nu$ as the projection of $Q$ onto the graph of $f$. For $A \subset G(f)$, let $\nu(A)=Q\{x:(x, f(x)) \in A\}$.

Given $\varepsilon>0$, pick $k$ so that $\left(n_{k+1}\right)^{-1} \leq \varepsilon<\left(n_{k}\right)^{-1}$. Since $\mid S_{k+1}(x)-$ $S_{k}(x)|=1,| S_{k+1}(x)-S_{k+1}(x(k+1)) \mid \leq 4 \pi$, and $S_{k}(x)$ approximates the slope on the interval, the projection of $\operatorname{Ball}((x, f(x)), \varepsilon)$ onto the $x$-axis yields an interval $I$ of diameter less than a constant multiple of $\varepsilon /\left|S_{k}(x)\right|$. Thus,

$$
\begin{aligned}
\frac{\nu\left(B_{\varepsilon}(x, f(x))\right)}{\varepsilon} & \leq \frac{Q\{\operatorname{Proj} \operatorname{Ball}((x, f(x)), \varepsilon)\}}{\varepsilon} \\
& \leq \frac{1}{\varepsilon} \sum_{j}\left\{Q\left(I_{k+2}(j)\right): \operatorname{Proj} \subset \bigcup_{j} I_{k+2}(j)\right\} .
\end{aligned}
$$

By Lemma 4, $Q\left(I_{k+2}(j)\right) \leq C \log (k+2) / n_{k+2}$ and the number of intervals of length $\left(n_{k+2}\right)^{-1}$ containing the projection, $I$, is less than $n_{k+2}|I|$ plus possibly two intervals for the ends. Consequently, the above is less than

$$
C\left(\frac{1}{\varepsilon}\right)\left(\frac{1}{n_{k+2}}\right) \log (k+2)\left(2+n_{k+2} \frac{C^{\prime} \varepsilon}{\left|S_{k}\right|}\right) \text {. }
$$

Since $\left|S_{k}\right|$ grows more rapidly than any power of $\log k$ via Theorem 1 , this tends to 0 as $k \rightarrow \infty \quad(\varepsilon \rightarrow 0) \quad Q$-a.e and so $\nu$-a.e.

Consider $h(x)=x / g\left(\log x^{-1}\right)$ where $g$ satisfies $\sum \log g\left(e^{e^{n}}\right) / e^{n}<\infty$ so that $Q\left\{\left|S_{n}\right|<g(n)\right.$ i.o. $\}=0$. Replacing $\varepsilon$ with $h(\varepsilon)$ in the denominator of the formula in the theorem of Rogers and Taylor, we see that $\nu\left[B_{\varepsilon}(x, f(x))\right] / h(\varepsilon)$ $\rightarrow 0$ as $\varepsilon \rightarrow 0 \quad \nu$-a.e. This implies via the same theorem of Rogers and Taylor that the graph of $f$ does not have $\sigma$-finite $m_{h}$-measure. Upper bounds are not as easy to obtain. One method might be to apply the good points-bad points arguments of Taylor but we have not yet succeeded in doing so.

\section{REFERENCES}

1. J. M. Anderson, E. A. Housworth, and L. D. Pitt, The spectral theory of multiplication operators and recurrence properties for nondifferentiable functions in the Zygmund class, $\Lambda_{a}^{*}$, Mathematika 39 (1992), 136-151.

2. J. M. Anderson and L. D. Pitt, Recurrence properties of certain lacunary series, J. Reine Angew. Math 377 (1987), 65-82.

3. Math. Soc. (3) 59 (1989), 558-592.

4. S. Axler, Harmonic functions from a complex analysis viewpoint, Amer. Math. Monthly 93 (1986), 246-258.

5. R. Durrett, Brownian motion and martingales in analysis, Wadsworth, Belmont, Calif., 1984.

6. A. Dvoretsky and P. Erdös, Some problems on random walk in space, Proc. 2nd Berkeley Sympos. Math. Stat. and Prob., Univ. of California Press, 1951, pp. 353-367.

7. W. Feller, An introduction to probability theory and its applications, Vol. II, Wiley, 1971.

8. J. Hawkes, Probabilistic behavior of some lacunary series, Z. Wahr. Verw. Gebiete 53 (1980), 21-33.

9. E. A. Housworth, Escape rates for a conditioned 2-dimensional Brownian motion and recurrence results for analytic Zygmund functions with applications, Dissertation, Univ. of Virginia, 1992. 
10. G. Hunt, Some theorems concerning Brownian motion, Trans. Amer. Math. Soc. 81 (1956), 294-319.

11. F. Knight, Brownian local times and taboo processes, Trans. Amer. Math. Soc. 143 (1969), 173-185.

12. Essentials of Brownian motion and diffusion, Math. Surveys, vol. 18, Amer. Math. Soc., Providence, R.I., 1981.

13. S. Kochen and C. Stone, A note on the Borel-Cantelli problem, Illinois J. Math. 8 (1964), 248-251.

14. R. D. Mauldin and S. C. Williams, On the Hausdorff dimension of some graphs, Trans. Amer. Math. Soc. 298 (1986), 793-803.

15. C. A. Rogers and S. J. Taylor, Functions singular and continuous with respect to a Hausdorff measure, Mathematika 8 (1961), 1-31.

16. T. Shiga and S. Watanabe, Bessel diffusions as a one-parameter family of diffusion processes, Z. Wahr. Verw. Gebiete 27 (1973), 37-46.

Department of Mathematics, Purdue University, West Lafayette, Indiana 47907

E-mail address: eah@math.purdue.edu 